\title{
Studium und Praktikum im Ausland
}

\author{
Fakten, Tipps und Hinweise
}

\section{Claudius Habbich}

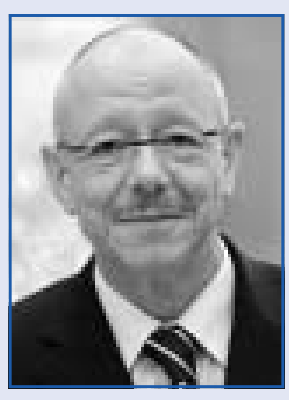

Claudius Habbich, M.A., war von 1997 bis Ende 2014 Leiter des Referats „Information für Deutsche über Studium und Forschung im Ausland; Publikationen" beim Deutschen Akademischen Austauschdienst (DAAD) in Bonn sowie Koordinator der Informations- und Werbekampagne "go out! studieren weltweit", die seit 2006 als gemeinsame Initiative vom Bundesministerium für Bildung und Forschung (BMBF) und vom DAAD mit zahlreichen Maßnahmen und Aktivitäten durchgeführt wird.

Ob während des (Bachelor-)Erststudiums, in einem Master-Studiengang oder zwischen zwei Ausbildungsabschnitten - ein Auslandsaufenthalt in Form eines zeitweiligen Auslandsstudiums, eines Praktikums, eines längeren Sprach- und Fachkurses oder in anderer Form dürfte fast immer persönlich und fachlich ein Gewinn sein, auf den Studierende nicht verzichten sollten. Und ein Pluspunkt im Lebenslauf ist er allemal!

Stichwörter: Studienbezogene Auslandsmobilität deutscher Studierender, Studienaufenthalte und Praktika im Ausland, Planung und Vorbereitung, Anerkennung im Ausland erbrachter Studienleistungen, Informations- und Kontaktstellen

\section{Ausgangspunkt: Studienbezogener Auslandsaufenthalt - ja oder nein?}

Die Frage wird Studienberatern in den Hochschulen oft gestellt: „Ich möchte mal eine Zeit im Ausland studieren was muss ich tun?" Studierende und Studieninteressierte müssen sich zunächst darüber klar werden, was für sie fachlich und persönlich wichtig ist und wie sie ihr Studium insgesamt planen. Kommt eine Studienphase im Ausland (für mich) überhaupt in Frage? Als Auslandssemester oder als längeres Studium im Ausland? Oder ist ein kürzerer studienbezogener Auslandsaufenthalt, beispielsweise ein Sprach- oder Fachkurs oder eine Studienreise, besser geeignet? Ist vielleicht ein Auslandspraktikum oder eine Recherche- bzw. Forschungsarbeit fachlich nützlich und eine inhaltliche Ergänzung des Curriculums? Mit welchem Ziel will ich wie lange an einer Hochschule im Ausland studieren? Wie passt der geplante Auslandsaufenthalt in den Stu- dienablauf? Sind Auslandsaufenthalte in der Studienordnung vorgesehen? Welche Hochschulkooperationen und Austauschprogramme hat die heimische Hochschule beziehungsweise der Fachbereich oder die Fakultät mit ausländischen Partnern? Viele Fragen, die Antworten in einem Entscheidungs- und Findungsprozess verlangen!

Optimal ist es, möglichst zu Beginn des Studiums oder vorher zu überlegen, ob ein Auslandssemester, ein Auslandsjahr oder ein Auslandspraktikum Teil des Studiums sein soll. Denn bereits mit der Wahl der Fachrichtung und des Studiengangs gibt es die Gelegenheit, gezielt nach Studiengängen Ausschau zu halten, in deren Curricula gegebenenfalls Auslandsphasen in obligatorischer Form oder optional eingefügt sind. Generell ist ein studienbezogener Auslandsaufenthalt ein Pluspunkt für jede spätere Bewerbung und die berufliche Karriere. Jedoch muss der Auslandsaufenthalt auch fachlich und zeitlich sinnvoll in das Studium integriert werden. Als Alternative kommt vielleicht auch ein Aufbau- oder Weiterbildungsstudium im Ausland nach dem Studienabschluss in Deutschland in Betracht.

Ein studienbezogener Auslandsaufenthalt trägt zur „Erweiterung des Horizonts“ und zur Weiterentwicklung der Persönlichkeit bei. Er bietet die Chance, Sachverhalte aus einer neuen Perspektive zu betrachten, fachlich und persönlich etwas hinzu zu lernen, die Sprachkompetenz vor Ort zu verbessern, interkulturelle Erfahrungen zu sammeln und internationale Kontakte zu knüpfen. Neugier und etwas Abenteuerlust gehören immer auch dazu.

\section{Auslandsmobilität deutscher Studierender während des Studiums}

\subsection{Anteile nach Hochschularten und Fächern}

Das Bundesministerium für Bildung und Forschung $(B M B F)$ und der Deutsche Akademische Austauschdienst $(D A A D)$ haben sich zum Ziel gesetzt, dass zukünftig mindestens jeder zweite Studierende studienbezogene Auslandserfahrungen während seines Studiums an einer Hochschule in Deutschland gemacht haben sollte. Verbunden ist das mit der Aufforderung an die Hochschulen, die Rahmenbedingungen für diese Mobilität zu gewährleisten. Viele Hochschulen haben bereits Auslandsphasen in Studiengänge integriert und wissen, dass die studienbezogene Auslandsmobilität deutscher Studierender ebenfalls ein Teil einer internationalen Ausrichtung ist und erheblich zur „Internationalität“ ihrer Einrichtung beiträgt. 
Zur Orientierung seien hier einige ausgewählte Daten zur temporären Auslandsmobilität genannt. Zu Beginn des Jahres 2013 hatten - auf Studierende aller Semester bezogen - insgesamt $26 \%$ der deutschen Studierenden in einem Erst- oder einem Masterstudium an einer deutschen Hochschule studienbezogene Auslandserfahrungen gemacht, wobei es Unterschiede bei den Hochschul- und Abschlussarten gibt. Die vom DAAD beim Deutschen Zentrum für Hochschul- und Wissenschaftsforschung (1) in Auftrag gegebene Studie ,Internationale Mobilität im Studium 2013“6 (2) resümiert als Ergebnis einer repräsentativen Befragung mit 14.000 in die Auswertung einbezogenen Antworten, dass die Studierenden in Bachelor-Studiengängen an Universitäten mit $16 \%$ weniger auslandsaktiv waren als ihre Kommilitonen an Fachhochschulen mit $19 \%$ - von den Master-Studierenden an Universitäten jedoch $39 \%$ über studienbezogene Auslandserfahrungen verfügten, gegenüber $33 \%$ der Studierenden in MasterStudiengängen an Fachhochschulen (jeweils bezogen auf die Studierenden aller Semester).

Von den Studierenden in Fächern mit Staatsexamen (z. B. Medizin, Pharmazie, Jura) hatten $24 \%$ derartige Erfahrungen gesammelt. Da in diesen Fächern eine längere Studienzeit vorgesehen ist, wachsen die Anteile auch mit der Zahl der Semester: von $22 \%$ im 5./6. Semester, auf $25 \%$ im 7./8. und $32 \%$ im 9./10. Semester - bei Studierenden im 11./12. Semester werden erstaunliche $49 \%$ erreicht, was allerdings auch mit Studienstrukturen und Fachkulturen in Zusammenhang zu bringen ist.

Bei den anderen Studiengängen ist ebenfalls zu beachten, dass der Anteil der Auslandserfahrenen mit der Anzahl der Semester wächst: Bei den Studierenden in BachelorStudiengängen beträgt er im 5./6. Semester an Universitäten $25 \%$ und an Fachhochschulen $21 \%$; dauert das Studium länger, so sind es im 7./8. Semester $29 \%$ an Universitäten und sogar $37 \%$ an Fachhochschulen. Entsprechend entwickeln sich auch die Anteile bei Studierenden im Master-Studium: Liegt hier der Anteil der Auslandserfahrenen im 1./2. Semester bereits bei $34 \%$ an Universitäten und $30 \%$ an Fachhochschulen, sind es im 3./4. Semester $39 \%$ (Universitäten) und $36 \%$ (Fachhochschulen) - bei längerem Studienverlauf haben im 5./6. Semester sogar $44 \%$ der Master-Studierenden an Universitäten und $37 \%$ an Fachhochschulen studienbezogene Auslandserfahrungen erworben.

Besonders ,auslandsmobil“ sind die Studierenden der Fachrichtungen im Bereich Wirtschaftswissenschaften gewesen, von denen $36 \%$ an Universitäten und $30 \%$ an Fachhochschulen im Ausland waren. Auch die Studierenden an Universitäten in Disziplinen der Sprach- und Kulturwissenschaften (33\%), der Sozialwissenschaften (31\%) und der Medizin (29\%) sowie der Agrar-, Forst und Ernährungswissenschaften an Fachhochschulen (28\%) können überdurchschnittliche Werte bei der Auslandsmobilität vorweisen.

$\mathrm{Zu}$ den weitaus weniger Auslandsaktiven gehören mit unterdurchschnittlichen Anteilen die Studierenden der Ma- thematik und Naturwissenschaften mit $20 \%$ Auslandserfahrenen in Universitäten und $12 \%$ in Fachhochschulen. Und auch die Studierenden der Ingenieurwissenschaften schneiden mit $19 \%$ Auslandsmobilen in universitären Studiengängen und mit $16 \%$ in entsprechenden Fächern an Fachhochschulen nicht viel besser ab.

Die Studie konstatiert als Ergebnis der vierten Studierendenbefragung im Projektbericht einen über mehrere Jahre währenden Bedeutungsverlust des Auslandspraktikums mit einem Anteil von $35 \%$ gegenüber $41 \%$ im Jahr 2007 und vermutet einen ,Zusammenhang mit den stark strukturierten Curricula der Bachelorstudiengänge ..., die auch in den Semesterferien weniger Raum für Praktika gewähren“ (Heublein/Ebert/Hutzsch/Richter/Schreiber, 2013, S. 10). Bei den Studierenden der Wirtschaftswissenschaften in Bachelor- und Master-Studiengängen beträgt der Anteil der Auslandspraktika unter den Arten der studienbezogenen Auslandsaufenthalte $29 \%$ an Universitäten und $31 \%$ an Fachhochschulen. Diese Anteile weichen bis auf Medizin (71\%) und Rechtswissenschaften (40\%) an Universitäten - nicht besonders wesentlich von denen in anderen Fächergruppen ab.

\subsection{Aufenthaltsdauer bei studienbezogenen Auslandsaufenthalten}

Der Aufenthaltszweck bestimmt ganz wesentlich die Dauer der Auslandszeit: Bei einem Auslandsstudium sind es durchschnittlich sieben Monate, wobei jeder zweite Aufenthalt fünf bis sechs Monate dauerte und ein Drittel sieben Monate oder länger (Median: sechs Monate). Für Praktika hielten sich Studierende im Durchschnitt vier Monate im Ausland auf, am Häufigsten drei Monate. $38 \%$ der Praktika dauerten bis zu zwei Monate, $26 \%$ drei bis vier Monate und $27 \%$ fünf bis sechs Monate, $9 \%$ sogar länger.

\subsection{Desinteresse an Studium und Praktikum im Ausland}

Tatsache ist, dass im Jahr 2013 rund $38 \%$ der Studierenden an Universitäten und $42 \%$ der Studierenden an Fachhochschulen kein Interesse an einem studienbezogenen Auslandsaufenthalt hatten - im Jahr 2007, bei der ersten repräsentativen Befragung waren es nur $29 \%$ an Universitäten und $40 \%$ an Fachhochschulen. Auch bei den Bachelor-Studierenden wuchs das generelle Desinteresse an Auslandsaufenthalten: von $29 \%$ auf $34 \%$ an Universitäten und von $37 \%$ auf $41 \%$ an Fachhochschulen. Bei den Fächern mit Staatsexamen schlossen $43 \%$ der Studierenden 2013 gegenüber $33 \%$ vor sechs Jahren ein Auslandsstudium, ein Auslandspraktikum oder eine andere Form des Auslandsaufenthalts während des Studiums für sich aus.

\subsection{Formen studienbezogener Auslandsaufenthalte}

Etliche Studierende gehen in unterschiedlichen Formen mehrfach ins Ausland, jeden zweiten zieht es zum Studium zeitweilig in die Ferne: Mit 55 \% war die Mehrheit der 
Studierenden an einem Auslandsstudium interessiert, $35 \%$ absolvierten - sowohl an Universitäten wie an Fachhochschulen - ein Auslandspraktikum, $12 \%$ machten eine Studienreise, $8 \%$ belegten einen Sprachkurs, $5 \%$ beteiligten sich an einer Sommerschule und $4 \%$ erstellten eine Projektarbeit im Ausland. Ferner gaben $5 \%$ der Befragten an, zu sonstigen Zwecken im Ausland gewesen zu sein. Studierende an Universitäten sind etwas häufiger zum Studium im Ausland als Studierende an Fachhochschulen, die wiederum zu einem leicht höheren Anteil Studienreisen ins Ausland machten, dort an Sommerschulen teilnahmen oder Projektarbeiten durchführten.

Der Anteil der Studierenden an Universitäten, die einen Auslandsaufenthalt zum zeitweiligen Studium an einer ausländischen Hochschuleinrichtung nutzten, ist am höchsten in Fachdisziplinen der Wirtschaftswissenschaften $(74 \%)$ - ein deutlicher Abstand zu den Anteilen, die sich bei den auslandserfahrenen Studierenden in den Sozialwissenschaften (62\%), aber auch in den Rechtswissenschaften $(58 \%)$ sowie im Fächerbereich von Sprach- und Kulturwissenschaften und Sport $(57 \%)$ und der Ingenieurwissenschaften $(57 \%)$ ergeben. Auch an den Fachhochschulen wurde in den wirtschaftswissenschaftlichen Fächern mit einem Anteil von fast $60 \%$ das Studium als bevorzugte Form des studienbezogenen Auslandsaufenthalts gewählt; die Anteile in anderen Fächergruppen fielen dagegen mit $35 \%$ (Mathematik/Naturwissenschaften) bis $40 \%$ und darüber (Sozialwesen, Ingenieurwissenschaften) nicht so hoch aus.

\subsection{Nutzen und Erträge der Auslandsmobilität}

Aus der Sicht der auslandsmobilen Studierenden gibt es eine ganze Reihe von Erträgen durch einen studienbezogenen Auslandsaufenthalt. Die Befragung zeigt eine Rangfolge auf, die stark durch Aspekte der Persönlichkeitsentwicklung und interkulturelle Orientierungen geprägt ist: Freundschaften zu Menschen aus anderen Ländern (19\%), Fähigkeit, mit neuartigen Situationen zurecht zu kommen (17\%), Verständnis anderer Arbeits- und Lebensweisen (17\%), Fremdsprachenkenntnisse (16\%), Selbständigkeit (9\%), Kenntnis eigener Stärken und Schwächen (8\%). Nachrangig scheinen dagegen für die Studierenden die fachlichen und beruflichen Aspekte eines studienbezogenen Auslandsaufenthalts zu sein, denn der Erwerb fachlicher Kenntnisse (4\%), Klarheit über berufliche Ziele (3\%), Vorbereitung auf eine zukünftige Berufstätigkeit (2\%), Klarheit über weitere Studienorientierung (2\%), beruflich relevante Kontakte (1\%) und die Entwicklung von Fähigkeiten zum wissenschaftlichen Arbeiten (1\%) spielen keine besondere Rolle in der Auflistung.

\subsection{Zielländer und Regionen}

Die deutschen Studierenden und Graduierten wollen für studienbezogene Auslandsaufenthalte in erster Linie ,nach Westen“: Bevorzugte Länder in Europa sind laut Studie Großbritannien (12\%), Frankreich (10\%) und Spanien (9\%). Mit Abstand folgen dann Schweden (6\%), Italien und die Schweiz (4\%) sowie Irland, die Niederlande, Österreich und Polen (je $3 \%$ ). Bei den außereuropäischen Ländern ist das Interesse nach wie vor an einem Aufenthalt in den USA (11\%) sowie den beiden englischsprachigen Ländern Australien und Kanada (je $3 \%$ ) am größten. Bemerkenswert ist allerdings, dass - wohl aufgrund vieler wissenschaftlicher Kooperationen und akademischer Austauschvereinbarungen - die VR China (4\%) als Zielland für Studienaufenthalte und Praktika noch etwas größere Bedeutung gewonnen hat.

Betrachtet man die Auslandsmobilität deutscher Studierender nach Regionen bleiben $60 \%$ von ihnen in Westeuropa (im Vergleich zu 2007: $-5 \%$ ), zieht es $14 \%(+1 \%)$ nach Nordamerika, $12 \%(+3 \%)$ in asiatische Länder und $11 \%(+1 \%)$ in Länder Osteuropas, $8 \%(+1 \%)$ entscheiden sich für Gastländer in Süd- und Mittelamerika, $6 \%$ $(+/-0 \%)$ für Afrika sowie $5 \%$ (-2\%) für Australien und Ozeanien.

\section{Planung und Vorbereitung von Auslandsaufenthalten}

Das A und $\mathrm{O}$ ist eine rechtzeitige und gezielte Vorbereitung mit sehr viel Informationsrecherche und Kontakten $\mathrm{zu}$ Informations- und Beratungsstellen, möglichen Gasthochschulen im Ausland und auslandserfahrenen Kommilitonen und Dozenten. Neben der notwendigen Ausdauer bei den Vorbereitungsschritten ist auch ein guter Zeitplan in Zusammenhang mit einer ,Checkliste“" (3) erforderlich, denn bis zu anderthalb Jahre sollten vom ersten Gedanken bis zur tatsächlichen Abreise in der Regel zeitlich veranschlagt werden. Vom Beschaffen der notwendigen Informationen bis hin zur Bewerbung an einer Hochschule im Ausland und um ein Stipendium oder in einem Förderungsprogramm ist schon in der ersten Phase mit einigen Monaten rechnen. Das Bearbeitungs- und Auswahlverfahren für ein Stipendium nach Ablauf der Bewerbungsfrist dauert je nach Art des Programms mehrere Wochen oder einige Monate. Nach einer erfolgreichen Bewerbung ist noch die konkrete Vorbereitung des Auslandsaufenthalts hinzuzurechnen.

Nachfragen zur späteren Anerkennung von im Ausland erbrachten Studienleistungen und Prüfungen sowie zu Einschreibungsvoraussetzungen an der Gasthochschule gehören ebenso dazu wie Aspekte der Unterkunft und der Lebensmodalitäten am Zielort. Ganz wichtig sind Sprachkenntnisse, denn je besser die Verständigung an der Hochschule und im Alltag möglich ist, desto erfolgreicher und nachhaltiger wird der Auslandsaufenthalt sein. Selbst wenn Kurse und Unterrichtsveranstaltungen für internationale Studierende auf Englisch angeboten werden, ist es sehr hilfreich, in der Landessprache kommunizieren $\mathrm{zu}$ können. Letztlich öffnet diese Fähigkeit viele Türen - und vor allem auch die Herzen der Menschen im Gastland!

Wer einen Auslandsaufenthalt während des Studiums realisieren möchte, sollte in mehreren, durchaus parallelen 
Schritten vorgehen: Informationen im Internet recherchieren, auslandserfahrene Kommilitonen und Dozenten befragen, thematisch einschlägige Veranstaltungen und Infobörsen besuchen, Informationsstellen an der eigenen Hochschule nutzen. Die wichtigste Kontaktstelle an der eigenen Hochschule ist das Akademische Auslandsamt bzw. das Internationale Büro/International Office. An vielen Hochschulen gibt es auch in den Fakultäten bzw. Fachbereichen zusätzlich Koordinatoren oder Beauftragte für internationale Beziehungen, die für Hochschulkooperationen und den akademischen Austausch zuständig sind. An zahlreichen Universitäten ist ferner ein ,Carrier Service“" vorhanden, der als Informations- und Beratungsstelle vor allem bei Praktika und zum Berufseinstieg vielfältige Angebote bereithält. Diese sollten jeweils erste Anlaufstelle für die verschiedensten Fragen sein. Dort werden die Studierenden beraten und erhalten Informationen über Stipendien, beispielsweise zum EU-Programm ,Erasmus+ “ oder zu $D A A D$-Stipendien, sowie zu anderen Förderungsprogrammen, zu den Möglichkeiten und Vorteilen, die Kooperationsvereinbarungen mit Partnerhochschulen im Ausland bieten, zu Bewerbungsvoraussetzungen, aber auch zu Terminen und zu Studienmöglichkeiten an Hochschulen im Ausland und zu Auslandspraktika. Darüber hinaus haben zahlreiche ausländische Botschaften und Kulturinstitute umfangreiche Informations- und Beratungsangebote - nicht nur auf Webseiten im Internet, sondern häufig auch in Räumen ihrer Einrichtungen in Deutschland.

Zum anderen hat der $D A A D$ ein großes Informationsangebot im Internet (www.daad.de), das Tipps zu Studium und Praktikum im Ausland zu allen Ländern der Welt mit Texten und Web-Links (4) ebenso bietet wie verschiedene Suchfunktionen zu internationalen Studiengängen (5) und Sprachkursen an Hochschulen im Ausland (6) sowie zu Stipendien und Förderungsmöglichkeiten (7). Viele dieser Informationen gibt es auch in Druckfassungen, die in den Akademischen Auslandsämtern/Internationalen Büros der Hochschulen zu erhalten sind.

\section{Anerkennung von im Ausland erbrachten Studienleistungen}

Viele Studierende klagen, dass sie Schwierigkeiten bei der Anerkennung von im Ausland erbrachten Studienleistungen hätten. Vor allem scheint es nach ihren Aussagen oftmals Unterschiede bei den Auskünften zwischen fachlich zuständigen Professoren bzw. Fachprüfern und den Prüfungsämtern der Hochschulen zu geben, deren Maßstäbe anscheinend an vorgegebenen Regularien ausgerichtet sind. Fast zwei Fünftel der Studierenden mit studienbezogenen Auslandsaufenthalten haben damit Probleme. Und für annähernd ein Drittel bedeutete der Auslandsaufenthalt deshalb einen Zeitverlust im Studium. Es ist besonders wichtig, dass Studierende die Anerkennungsaspekte bei der Planung und Vorbereitung des Auslandsaufenthalts mit den Zuständigen, z. B. mit den Austauschkoordinatoren und den Prüfungsbeauftragten, nicht nur besprechen, son- dern im Detail klären. Zur Anerkennung von im Ausland erbrachten Studienleistungen haben die meisten deutschen Hochschulen ein Verfahren vorgesehen, bei dem durch den Austauschkoordinator und den Studierenden gemeinsam ein (persönliches), ,Learning Agreement“" erstellt wird, das anschließend zusammen mit einem ,Transcript of Records“ (Notenabschrift/Zeugnis der Hochschule im Ausland) die Grundlage für den Anerkennungsbescheid ist. Informationen dazu sind im Web unter www.daad.de $>$ Infos für Deutsche (8) zu finden.

\section{Stipendien und Förderungsmöglichkeiten}

Bei der Finanzierung von studienbezogenen Auslandsaufenthalten gibt es unterschiedliche Möglichkeiten und Unterstützungsformen, die hochschulspezifisch ausgeprägt sein können. Wegen der Vielfalt und Differenziertheit der Programme ist es notwendig, sich über die jeweiligen Voraussetzungen und organisatorischen Rahmenbedingungen zu informieren. Viele Austauschprogramme und Stipendien sind fachspezifisch oder regional ausgerichtet oder haben andere Bedingungen, die den Bewerberkreis jeweils eingrenzen.

Die Palette in Frage kommender Finanzierungsquellen für studienbezogene Auslandsaufenthalte erstreckt sich von Stipendien und strukturierten Förderungsprogrammen des $D A A D$ (9), anderer Organisationen und ausländischer Regierungen über Programme der Bundesländer, der deutschen Hochschulen und der Europäischen Union (,,Erasmus+ “) bis hin zu Auslands-BAföG und Bildungskrediten zu günstigen Bedingungen (10). Auch Hochschulpartnerschaften bieten gegebenenfalls erhebliche Vergünstigungen und können zur finanziellen Entlastung beitragen.

\section{Zusammenfassung}

Ein studienbezogener Auslandsaufenthalt ist ein „Plus“ im Lebenslauf, denn er belegt die Bereitschaft, sich mit einer neuen, andersartigen und kulturell verschiedenen Umgebung durch persönliche Erfahrung auseinanderzusetzen. Neben dem Erwerb von fachlichen Kenntnissen an einem anderen Ort sind es gerade die sprachlichen Kenntnisse, die sozialen Kompetenzen und andere Fähigkeiten, die dabei entwickelt und erweitert werden können. In einer ,globalisierten Welt", die in gesellschaftlichen Zusammenhängen, besonders in Wirtschaft, Wissenschaft und Kultur zukünftig immer stärker vernetzt sein wird, sind derartige Erfahrungen während des Studiums ein großer Gewinn für den weiteren Berufs- und Lebensweg. Das wird auch bei Personalentscheidungen beziehungsweise bei Bewerbungen gesehen und kann als Merkmal durchaus ausschlaggebend sein.

\section{Anmerkungen und Hinweise zur Recherche}

Die Webseite www.go-out.de der Kampagne zur internationalen Mobilität deutscher Studierender ,go out! studieren weltweit“ wurde zum Ende des Monats November 2015 abgeschaltet und im Rah- 
men eines ,Relaunchs“ der Informations- und Werbekampagne durch die Domain www.studieren-weltweit.de ersetzt.

(1) Das HIS-Institut für Hochschulforschung der HIS-Hochschul-Informations-System $\mathrm{GmbH}$ (Hannover) ist die Vorgänger-Institution des DZHW - Deutsches Zentrum für Hochschul- und Wissenschaftsforschung $\mathrm{GmbH}$, die im August 2013 neu gegründet wurde; Gesellschafter sind die Bundesrepublik Deutschland und die Bundesländer.

(2) DAAD, Deutscher Akademischer Austauschdienst/HIS-Institut für Hochschulforschung, Internationale Mobilität im Studium 2013. Ergebnisse der vierten Befragung deutscher Studierender zu studienbezogenen Aufenthalten im Ausland, Projektbericht, Hannover, September 2013; Ausgewählte Ergebnisse der 4. Befragung deutscher Studierender zu studienbezogenen Aufenthalten in anderen Ländern 2013, präsentiert auf der 7. Fachkonferenz ,go out! studieren weltweit“ zur Auslandsmobilität deutscher Studierender, Mai 2013, S. 7-8, Online im Internet: www.dzhw.eu $>$ Publikationen $>$ Projektberichte

(3) www.daad.de $>$ Infos für Deutsche $>$ Studieren im Ausland $>$ Planung und Bewerbung $>$ Checkliste zum Auslandsstudium (PDF zum Download)

(4) www.daad.de $>$ Infos für Deutsche (dort u.a.) $>$ Länder A-Z

(5) www.daad.de $>$ Infos für Deutsche $>$ Studieren im Ausland $>$ Studieren und Leben $>$ Internationale Studiengänge (www. daad.de/ausland/studieren/leben/de/97-internationale-studiengaenge/)

(6) www.daad.de $>$ Infos für Deutsche $>$ Sprachen lernen $>$ Sprachkurse an Hochschulen in ausgewählten Ländern (www.daad. de/ausland/sprachen-lernen/sprachkurse/de/476-sprachkursean-hochschulen-in-ausgewaehlten-laendern/)

(7) www.daad.de $>$ Infos für Deutsche $>$ Stipendien finden und bewerben (www.daad.de/ausland/studieren/stipendium/de/ 70-stipendien-finden-und -bewerben/)
(8) www.daad.de >Infos für Deutsche $>$ Studieren im Ausland $>$ Planung und Bewerbung >Anerkennung von Leistungen und Abschlüssen

(9) Habbich, C., Ohne Moos nix los! Studienaufenthalte im Ausland finanzieren, Teil 2: Förderungsprogramme für Hochschulen, in: WiSt - Wirtschaftswissenschaftliches Studium, 44. Jg. (2015), S. 218-221.

(10) Habbich, C., Ohne Moos nix los! Studienaufenthalte im Ausland finanzieren, Teil 1: Individualstipendien und andere $\mathrm{Fi}$ nanzierungsmöglichkeiten für Studierende und Graduierte, in: WiSt - Wirtschaftswissenschaftliches Studium, 44. Jg. (2015), S. 165-168.

\section{Literatur}

Heublein, U., J. Ebert, C. Hutzsch, J. Richter, J. Schreiber, Internationale Mobilität im Studium 2013, Projektbericht mit Ergebnissen der vierten Befragung deutscher Studierender zu studienbezogenen Aufenthalten im Ausland, Deutscher Akademischer Austauschdienst (DAAD), Bonn, Deutsches Zentrum für Hochschul- und Wissenschaftsforschung GmbH (DZHW), Hannover, September 2013.

DAAD, Deutscher Akademischer Austauschdienst/Deutsches Komitee der IAESTE (International Association for the Exchange of Students for Technical Experience)/IJAB-Fachstelle für Internationale Jugendarbeit der Bundesrepublik Deutschland/eurodesk Deutschland/GIZ, Deutsche Gesellschaft für Internationale Zusammenarbeit/ZAV, Zentrale Auslands- und Fachvermittlung der Bundesagentur für Arbeit, Wege ins Auslandspraktikum (planen-realisieren-finanzieren), Bonn 2013, Online im Internet: URL: www.daad.de >Infos für Deutsche>Praktika im Ausland $>$ Literatur $>$ Wege ins Auslandspraktikum (PDF) (www.daad.de/medien/ausland/dokumente/wege_ins_auslandspraktikum_2013.pdf).

\section{Die effiziente Jobsuche - so geht's einfach und schnell}

Jeder, der nach einer neuen beruflichen Herausforderung sucht, möchte sicherlich alles richtig machen und seine Möglichkeiten voll ausschöpfen, um den ersehnten Traumjob zu finden. Aber wie geht man dieses Ziel am besten an? Welche Wege lassen sich bei der Jobsuche bestreiten? Und wie kann die Suche möglichst zeiteffizient gestaltet werden?

Im ersten Schritt ist festzulegen, wonach man eigentlich sucht. Hilfreich ist, die eigenen Ziele zu definieren. Bei der Jobsuche gibt es viele Variablen zu beachten: Welche Ausbildung, Qualifikation und Fähigkeiten müssen für eine bestimmte Tätigkeit mitgebracht werden? Zudem sollte man für sich klären, ob für den neuen Job ein Umzug in eine andere Stadt oder in ein anderes Bundesland in Betracht kommt. Ebenso sollte darüber nachgedacht werden, ob man in der bisherigen Branche bleiben oder zukünftig gerne in einer anderen arbeiten möchte. Detailsuchfunktionen von Jobbörsen können helfen, eine passgenaue Jobsuche zu starten.

Es ist empfehlenswert, entsprechend der definierten Ziele ein Suchprofil anzulegen. Bei vielen Jobbörsen erhält man so täglich, wöchentlich oder monatlich aktuelle Stellenausschreibungen per E-Mail zugeschickt, die dem Suchprofil entsprechen und die geäußerten Wünsche berücksichtigen. Dies ermöglicht eine zeitnahe Reaktion auf interessante Angebote.
Die Bewerbungsunterlagen sollten dabei bereits im Vorfeld zusammengestellt worden sein. Befragungen ergaben, dass rund $75 \%$ der Personalverantwortlichen eine E-MailBewerbung bevorzugen. Für Bewerber ist dies durchaus positiv: Online-Bewerbungen sind deutlich kostengünstiger und schneller umsetzbar als postalisch versendete Bewerbungsmappen. Jobbörsen stellen neben Suchfunktionen häufig auch Tipps zur Verfügung, die bei der Erstellung und Aktualisierung von Bewerbungsunterlagen nützlich sein können.

Bewerber sollten zusätzlich erwägen, im Netz ein professionelles Karriereprofil anzulegen. Auf vielen Portalen lässt sich schnell und kostenlos der eigene Online-Lebenslauf hinterlegen - mit den wichtigsten Daten, erreichten Qualifikationen und allen Anhängen, die genauere Auskunft über die bisherige berufliche Laufbahn geben. Personalverantwortliche, die auf der Suche nach Mitarbeitern mit bestimmten Fähigkeiten sind, können so leicht geeignete Kandidaten finden und diese direkt kontaktieren.

\section{Literatur}

StepStone, Die effiziente Jobsuche - so geht's einfach und schnell, 2015, Online im Internet: URL: http://www.stepstone.de/Karriere-Bewerbungstipps/die-effiziente-jobsuche.cfm (Abrufdatum: 17.08.2015).

Felix Horstmann, M.Sc., Marburg 